\title{
ON PRINCIPAL LINES AND PRINCIPAL POINTS
}

\author{
SHU-CHU FONG
}

Recently Chang ${ }^{1}$ has enriched the contact theory of two plane curves by virtue of many projective correspondences. The object of this paper is to establish some analogous results regarding the contact theory of two space curves.

In an appendix to Fubini and Cech's book ${ }^{2}$ Bompiani remarked that the contact theory of two space curves can be treated by considering a double ratio analogous to that of C. Segre. ${ }^{3}$ Accordingly $\mathrm{Pa}-$ lozzi ${ }^{4}$ has obtained principal lines and principal points associated with two space curves which are tangent but have distinct osculating planes or possess a contact of higher order at an ordinary point. But in the latter case Stouffer ${ }^{5}$ has shown that neither principal lines nor principal points exist when the principal plane becomes the common osculating plane of the two curves. Naturally in Stouffer's case we should require new covariant figures. We solve this problem by obtaining a new covariant line.

Between the points $P$ and $\bar{P}$ of the two space curves $C$ and $\bar{C}$, which are tangent or intersect at an ordinary point, we define a correspondence $G$, such that the tangents to $C$ and $\bar{C}$ at corresponding points of $G$ intersect. This correspondence plays an important role in this paper because the joins of the corresponding points of $G$ constitute developables. We can deduce from $G$ new definitions of Bompiani's ${ }^{6}$ principal lines and principal points for two intersecting space curves.

Two space curves $C$ and $\bar{C}$ intersecting at an ordinary point $O(0,0,0)$ with distinct osculating planes may be represented by the

Received by the editors December 10, 1945, and, in revised form March 8, 1946, and June 13, 1946.

${ }^{1}$ Chang, $A$ new definition of Bompiani line and its generalization, in the hands of The International Cultural Service of China.

2 Fubini-Čech, Geometria proiettiva Differenziale, vol. 2, 1926.

${ }^{3}$ C. Segre, Sugli elementi curvilinea che hanno comme la tangente e il piano osculatore, Rendiconti della Classe di Scienze Fisiche, Matematiche e Naturali, R. Accademia Nazionale dei Lincei (5) vol. 33 (1924) pp. 325-329.

" Palozzi, Surgli invarianti proiettivi di contratto fra curve sghembe, Rendiconti della Classe di Scienze Fisiche, Matematiche e Naturali, R. Accademia Nazionale dei Lincei (6) vol. 7 (1928) pp. 321-325.

${ }^{5}$ Stouffer, On the contact of two space curves, Bull. Amer. Math. Soc. vol. 38 (1932) pp. 415-419.

- Bompiani, Invarianti d'intersezione di due curve sghembe, Rendiconti della Classe di Scienze Fisiche Matimatiche e Naturali, R. Accademia Nazionale dei Lincei (6) vol. 11 (1931) pp. 456-461. 
expansions

$$
\begin{aligned}
& y=a x^{3}+b x^{4}+\cdots, z=r x^{2}+s x^{3}+\cdots, \\
& x=\bar{a} y^{3}+b y^{4}+\cdots, z=\bar{r} y^{2}+s y^{3}+\cdots,
\end{aligned}
$$

respectively, where $x, y, z$ are nonhomogeneous coordinates of a point in space. After denoting the coordinates of the corresponding points $P$ and $\bar{P}$ by $(x, y, z)$ and $(\bar{x}, \bar{y}, \bar{z})$, where

$$
\bar{x}=\bar{a} \bar{y}^{3}+b \bar{y}^{4}+\cdots
$$

and

$$
\bar{z}=\bar{r} \bar{y}^{2}+\tilde{s} \bar{y}^{3}+\cdots,
$$

we easily see from the vanishing of the determinant

$$
\left|\begin{array}{cccc}
x & y & z & 1 \\
1 & d y / d x & d z / d x & 0 \\
\bar{x} & \bar{y} & \bar{z} & 1 \\
d \bar{x} / d \bar{y} & 1 & d \bar{z} / d \bar{y} & 0
\end{array}\right|
$$

that the correspondence $G$ is given by

$$
x=\tau_{1} \bar{y}+\tau_{2} \bar{y}^{2}+\cdots,
$$

where

$$
\tau_{1}= \pm\left(\frac{\bar{r}}{r}\right)^{1 / 2}, \quad \tau_{2}=\frac{s \tau_{1}^{3}-\xi}{-r \tau_{1}}
$$

The line $P \bar{P}$ has the equation

$$
\frac{X-x}{\bar{x}-x}=\frac{Y-y}{\bar{y}-y}=\frac{Z-z}{\bar{z}-z},
$$

which evidently approaches the principal line ${ }^{7}$

$$
X=-\tau_{1} Y, \quad Z=0
$$

as $x \rightarrow 0, X, Y, Z$ being the current coordinates of a point. In addition, the edges of regression of the developables constituted by the lines (6) pass through

(8) $\quad\left(\frac{\bar{r} \tau_{1}}{-\bar{s}+s \tau_{1}^{3}}, \frac{-\bar{r}}{-\bar{s}+s \tau_{1}^{3}}, 0,1\right), \quad\left(\tau_{1}= \pm\left(\frac{\bar{r}}{r}\right)^{1 / 2}\right)$,

\footnotetext{
${ }^{7}$ Bompiani, loc. cit.
} 
respectively. We have, therefore, reached the following theorem.

THeORem 1. When $C$ and $\bar{C}$ intersect at an ordinary point $O$ with distinct tangents, there are two and only two developables containing both $C$ and $\bar{C}$. The edges of regression of these two developables are tangent to the two principal lines at the two principal points.

Let $M$ be a moving point on $C$. We denote the two edges of regression in the above theorem by $E_{11}$ and $E_{12}$. If the tangents of $E_{11}$ and $E_{12}$ at their points $M_{11}$ and $M_{12}$ pass through $M$, then the line $M_{11} M_{12}$ constitutes a developable with edge of regression $E_{21}$ as $M$ varies on $C$. Similarly we obtain $E_{22}$ by substituting $\bar{C}$ for $C$. Clearly on $E_{2 i}$ $(i=1,2)$ there is a point $M_{2 i}$ at which the tangent to $E_{2 i}$ passes through $M_{11}$ and the line $M_{21} M_{22}$ constitutes a developable with edge of regression $E_{31}$ when $M_{11}$ varies on $E_{11}$. Thus we obtain $E_{k i}$ $(k=1,2,3, \cdots ; i=1,2)$. For brevity we denote $E_{k 1}$ and $E_{k 2}$ by $\left(E_{k}\right)$ and call $\left(E_{k}\right)$ the $G$-transform of $\left(E_{k-1}\right)$.

Since $G$ is one-to-two, a point $P$ on $C$ corresponds to two points $\bar{P}_{1}$ and $\bar{P}_{2}$ on $\bar{C}$. We denote the locus of the points of intersection $N_{11}$ and $N_{12}$ between the tangent of $C$ at $P$ and those of $\bar{C}$ at $\bar{P}_{1}$ and $\bar{P}_{2}$ by $F_{11}$ and $F_{12}$. When $P$ moves along $C$, the point of intersection of the tangents of $F_{11}$ and $F_{12}$ at $N_{11}$ and $N_{12}$ is a new curve $F_{21}$. The interchange between $\bar{C}$ and $C$ gives $F_{22}$. In this way we obtain the curves $F_{k i}(k=1,2, \cdots ; i=1,2)$, where $\left(F_{k}\right)$, which denotes the two curves $F_{k 1}$ and $F_{k 2}$, is evidently the $G$-transform of $\left(F_{k+1}\right)$.

THEOREM 2. With regard to the curves (1) and (2) there is an infinite sequence of pairs of curves

$$
\cdots,\left(F_{k}\right),\left(F_{k-1}\right), \cdots,\left(F_{1}\right),(C, \bar{C}),\left(E_{1}\right),\left(E_{2}\right), \cdots,\left(E_{k}\right), \cdots
$$

including the pair $C$ and $\bar{C}$ such that every pair is the G-transform of the preceding pair.

In case the osculating planes of $C$ and $\bar{C}$ at $O$ coincide, the expansions (1) and (2) should be replaced by

$$
y=a x^{2}+b x^{3}+\cdots, \quad z=r x^{3}+s x^{4}+\cdots,
$$

and

$$
x=\bar{a} y^{2}+\bar{b} y^{3}+\cdots, \quad z=\bar{r} y^{3}+\tilde{s} y^{4}+\cdots,
$$

respectively. Now (4) becomes

$$
x=\mu_{1} \bar{y}+\mu_{2} \bar{y}^{2}+\cdots
$$

where 


$$
\mu_{1}^{3}=\frac{\bar{r}}{r}, \quad \mu_{2}=\frac{1}{2 r \mu_{1}^{2}}\left(-s \mu_{1}^{4}+a \bar{r} \mu_{1}^{2}+\tilde{s}-\bar{a} r \mu_{1}^{2}\right) .
$$

Here the correspondence $G$ is one-to-three. Clearly the joins of the corresponding points of $G$ approach principal lines when $P$ approaches $O$ and constitute three developables whose edges of regression $E_{1 k}$ $(k=1,2,3)$ pass through the three principal points respectively.

With respect to two roots of $\mu_{1}^{3}=\bar{r} / r$ we obtain through (11) two correspondences between points of $C$ and $\bar{C}$. In virtue of them we may determine a sequence of pairs of curves

$$
\begin{aligned}
\cdots,\left(F_{k}^{(l)}\right), \cdots,(C, \bar{C}), \cdots, & \left(E_{k}^{(l)}\right), \cdots, \\
& (k=1,2, \cdots ; l=1,2,3),
\end{aligned}
$$

analogous to that of Theorem 2. Consequently we obtain a total of three sequences of transforms.

We call the point of contact of the join $P \bar{P}$ and $E_{1 l}$ the corresponding point of $P$ or $\bar{P}$ on $E_{1 l}$. We have arrived at the following theorem.

ThEOREM 3. When $C$ and $\bar{C}$ intersect at an ordinary point $O$ with distinct tangents but common osculating plane, there are three developables passing through $C$ and $\bar{C}$ and three sequences of $G$-transforms (13). In particular the points corresponding to $O$ on the G-transforms of $C$ and $\bar{C}$ are the three principal points.

If $C$ and $\bar{C}$ have a common tangent at $O$, we take it for the $x$-axis. The expansions of $C$ and $\bar{C}$ now become

$$
y=x^{2} \sum_{0}^{\infty} a_{i} x^{i}, \quad z=x^{2} \sum_{0}^{\infty} b_{i} x^{i}
$$

and

$$
y=x^{2} \sum_{0}^{\infty} \bar{a}_{i} x^{i}, \quad z=x^{2} \sum_{0}^{\infty} \bar{b}_{i} x^{i} .
$$

If the osculating planes at $O$ of $C$ and $\bar{C}$ are distinct, the correspondence $G$ is analytically given by

$$
x=\bar{x}+m \bar{x}^{2}+n \bar{x}^{3}+\cdots,
$$

where

$$
m=\frac{1}{2\left(a_{0} \bar{b}_{0}-\bar{a}_{0} b_{0}\right)}\left[\left(b_{0}-\bar{b}_{0}\right)\left(a_{1}-\bar{a}_{1}\right)-\left(a_{0}-\bar{a}_{0}\right)\left(b_{1}-\bar{b}_{1}\right)\right],
$$




$$
\begin{aligned}
n= & -m^{2}-\frac{m}{\left(a_{0} b_{0}-\bar{a}_{0} b_{0}\right)}\left[2\left(a_{0} b_{1}-a_{1} b_{0}\right)+3\left(a_{1} b_{0}-\bar{a}_{0} b_{1}\right)\right. \\
& \left.+\left(a_{0} b_{1}-\bar{a}_{1} b_{0}\right)\right] \\
& +\frac{1}{a_{0} \bar{b}_{0}-\bar{a}_{0} b_{0}}\left[\left(a_{2}-\bar{a}_{2}\right)\left(b_{0}-\bar{a}_{0}\right)-\left(a_{0}-\bar{a}_{0}\right)\left(b_{2}-\bar{b}_{2}\right)\right] .
\end{aligned}
$$

Clearly the limit of the join of the corresponding points $P$ and $\bar{P}$ when $P \rightarrow O$ is the principal line ${ }^{8}$

$$
\begin{aligned}
& Y=\frac{a_{0}-\tilde{a}_{0}}{m} X, \\
& Z=\frac{b_{0}-\bar{b}_{0}}{m} X .
\end{aligned}
$$

Since the Halphen plane ${ }^{9}$ osculates the edge of regression of the developable consisting of the joins $P \bar{P}$ at the principal point, ${ }^{10}$ we have proved the following theorem.

Theorem 4. There is a unique developable passing through two space curves which are tangent at an ordinary point with distinct osculating planes. The edge of regression of this developable passes through the principal point, is tangent to the principal line, and osculates the Halphen plane.

Now the sequence of $G$-transforms of $C$ and $\bar{C}$ are terminated on both sides. Both $E_{1}$ and $F_{1}$ contain one curve only. In particular, $F_{1}$ has at $O$ a point of inflexion ${ }^{11}$ with the hyperosculating plane

$$
\frac{b_{0} Y-a_{0} Z}{\bar{b}_{0} Y-\bar{a}_{0} Z}=\frac{a_{0} b_{1}-a_{1} b_{0}}{\bar{a}_{0} b_{1}-\bar{a}_{1} b_{0}}
$$

which is a new covariant plane.

If $C$ and $\bar{C}$ have at $O$ contact of order $k+2$, then (14) and (15) become

$$
y=x^{2} \sum_{0}^{\infty} a_{i} x^{i}, \quad z=x^{3} \sum_{0}^{\infty} b_{i} x^{i}
$$

and

8 Palozzi, loc. cit.

- Palozzi, loc. cit.

10 Palozzi, loc. cit.

1 Chang, Contributions to projective theory of singular points of space curves, in the hands of The International Cultural Service of China. 


$$
y=x^{2} \sum_{0}^{\infty} \bar{a}_{i} x^{i}, \quad z=x^{3} \sum_{0}^{\infty} \bar{b}_{i} x^{i}
$$

where

$$
a_{l}=\tilde{a}_{l} \quad(l=0,1, \cdots, k) ; \quad b_{m}=b_{m} \quad(m=0,1, \cdots, k-1) .
$$

Here either $a_{k+1} \neq \bar{a}_{k+1}$ or $b_{k} \neq \bar{b}_{k}$ must be true. By a little consideration of the transformation

$$
x=\xi, \quad y=\eta, \quad \zeta=\left(b_{k}-b_{k}\right) y+\left(a_{k+1}-\bar{a}_{k+1}\right) z,
$$

we find two cases are essential to consider: firstly, $a_{k+1}=\bar{a}_{k+1}, b_{k} \neq b_{k}$ and secondly, $a_{k+1} \neq \bar{a}_{k+1}, b_{k}=\bar{b}_{k}$. In the latter case the Halphen plane and $Z=0$ coincide but in the former they do not.

For the case $b_{k} \neq \bar{b}_{k}, a_{k+1}=\bar{a}_{k+1}$ the correspondence $G$ may be analytically given by

$$
x=\bar{x}+\sum_{\nu=k+2}^{\infty} C_{\nu} \bar{x}^{\nu+1},
$$

where

$$
\begin{aligned}
C_{k+2}= & -\frac{1}{2 a_{0}}\left(a_{k+2}-\bar{a}_{k+2}\right) \\
C_{k+3}= & \frac{1}{2 a_{0}\left(b_{k}-b_{k}\right)}\left(a_{k+2}-\bar{a}_{k+2}\right)\left(b_{k+1}-\bar{b}_{k+1}\right) \\
& +\frac{3 a_{1}(k+2)}{4 a_{0}^{2}}\left(a_{k+2}-\bar{a}_{k+2}\right)-\frac{a_{k+3}-\bar{a}_{k+3}}{a_{0}} .
\end{aligned}
$$

After a little consideration we can prove the following theorem.

THEOREM 5. If $C$ and $\bar{C}$ have at $O$ contact of order $k+2(k \geqq 1)$ and if $a_{k+1}=\bar{a}_{k+1}, b_{k} \neq \bar{b}_{k}$, then the edge of regression $E$ of the unique developable containing $C$ and $\bar{C}$ passes through the principal point $T$. Furthermore, the tangent of $E$ at $T$ is the principal line and the osculating plane of $E$ at $T$ coincides with the Halphen plane of $C$ and $\bar{C}$.

If in the last two paragraphs we put $k=0$, then the correspondence $G$ is one-to-four and (21) should be replaced by

$$
x=\alpha_{i} \bar{x}+C_{2}\left(\alpha_{i}\right) \bar{x}^{2}+C_{3}\left(\alpha_{i}\right) \bar{x}^{3}+\cdots \quad(i=1,2,3,4),
$$

where $\alpha_{i}(i=1,2,3,4)$ are the four roots of the biquadratic equation

$$
b_{0} t^{4}-4 b_{0} t^{3}+\left(3 b_{0}+3 \bar{b}_{0}\right) t^{2}-4 \bar{b}_{0} t+\bar{b}_{0}=0 .
$$


It is very easy to prove the following theorem.

Theorem 6. If $C$ and $\bar{C}$ have at $O$ contact of order two where $b_{i} \neq \bar{b}_{0}$, then there are four developables passing through $C$ and $\bar{C}$. Only one of these four developables is not tangent to the plane $Z=0$ but tangent to the Halphen plane of $C$ and $\bar{C}$ along the principal line. The edge of regression of this particular developable passes through the principal point.

By virtue of every two correspondences of (23) we can derive a sequence of $G$-transforms associated with $C$ and $\bar{C}$. Therefore we have six sequences.

Now we consider the special case that $C$ and $\bar{C}$ are tangent and that their osculating planes at the point of contact are coincident. The expansions of $C$ and $\bar{C}$ are (19) and (20) where $a_{0} \neq \bar{a}_{0}$. The correspondence $G$ is also one-to-four and may be expressed by

$$
x=\beta_{i} \bar{x}+D_{2}\left(\beta_{i}\right) \bar{x}^{2}+D_{3}\left(\beta_{i}\right) \bar{x}^{3}+\cdots \quad(i=1,2,3,4),
$$

where $\beta_{i}(i=1,2,3,4)$ are the roots of

$$
a_{0} b_{0} t^{4}-4 \bar{a}_{0} b_{0} t^{3}+3\left(\bar{a}_{0} b_{0}+a_{0} \bar{b}_{0}\right) t^{2}-4 a_{0} b_{0} t+\bar{a}_{0} b_{0}=0 .
$$

In general $t \neq 1$ and we arrive at the following theorem.

THEOREM 7. There are four developables passing through the two space curves (19) and (20) which are tangent at an ordinary point with common osculating plane.

Naturally we can derive six sequences of curves by $G$-transforms. In general, all of these curves pass through $O$ and possess common tangent $Y=0, Z=0$ and common osculating plane $Z=0$.

In the last three paragraphs $b_{0} \neq b_{0}$. If the projecting cones of $C$ and $\bar{C}$ with common vertex on $Z=0$ have contact of third order, we have

$$
b_{0}=\bar{b}_{0} \text {. }
$$

Since $a_{0} \neq \bar{a}_{0}$, a single root of (25) is 1 , and one of the expressions (24) becomes

$$
x=\bar{x}-\frac{b_{1}-\bar{b}_{1}}{3 b_{0}} \bar{x}^{2}+\cdots .
$$

The developable consisting of the joins of the points of $C$ and $\bar{C}$ corresponding under (26) is tangent to $Z=0$ along the generator

$$
Z=0, \quad \frac{X}{b_{1}-\bar{b}_{1}}+\frac{Y}{3 b_{0}\left(a_{0}-\bar{a}_{0}\right)}=0 .
$$


Furthermore, the edge of regression of this developable intersects $Z=0$ at a new covariant point.

The plane $Z=0$ intersects the developables of $C$ and $\bar{C}$ in two plane curves

$$
y=\frac{3}{4} a_{0} x^{2}+\frac{3}{8} \frac{a_{0} b_{1}}{b_{0}} x^{3}+\cdots, \quad z=0
$$

and

$$
y=\frac{3}{4} \bar{a}_{0} x^{2}+\frac{3}{8} \frac{\bar{a}_{0} \bar{b}_{1}}{\bar{b}_{0}} x^{3}+\cdots, \quad z=0,
$$

which have at $O$ contact of order one and, therefore, possess a covariant line $r_{0}{ }^{12}$ which is coincident with (27). Hence we have reached the following theorem.

THEOREM 8. If on any plane through $Y=0, Z=0$ the projections of the curves (19) and (20) from any point on $Z=0$ have at $O$ contact of order three, one of the four developables mentioned in Theorem 7 does not pass through $Y=0, Z=0$ but through the covariant line $r_{0}$ of the curves (28) and (29).

If $b_{0} \neq \bar{b}_{0}$, the $r_{0}$ of (28) and (29) is found to be

$$
Z=0, \quad 3\left(\bar{a}_{0}-a_{0}\right) X+\left(\frac{\bar{b}_{1}}{\bar{b}_{0}}-\frac{b_{1}}{b_{0}}\right) Y=0,
$$

which is an important covariant line associated with the curves (19) and (20).

Now we deal with the remaining case that the coefficients in (19) and (20) are connected by the relations

$$
a_{m}=\bar{a}_{m}, \quad b_{m}=\bar{b}_{m} \quad(m=0,1, \cdots, k),
$$

where $a_{k+1} \neq \bar{a}_{k+1}(k \geqq 0)$. (21) should be replaced by

$$
x=\bar{x}+\sum_{\nu=k+1}^{\infty} D_{\nu} \bar{x}^{\nu+1},
$$

where $D_{k+1+\tau}=D_{k+1+\tau}\left(D_{k+1}\right)(\tau>0)$ and

$$
\begin{aligned}
D_{k+1}[ & \left.6 b_{0}\left(a_{k+1}-\bar{a}_{k+1}\right)-2 a_{0}\left(b_{k+1}-\bar{b}_{k+1}\right)\right] \\
+\left(a_{k+1}-\bar{a}_{k+1}\right)\left(b_{k+1}-\bar{b}_{k+1}\right) & =0 .
\end{aligned}
$$

12 Bompiani, loc. cit. Chang, loc. cit. footnote 1. 
Consequently we have proved the following theorem.

THEOREM 9. If $C$ and $\bar{C}$ have at $O$ contact of order $k+2(k \geqq 0)$ where $a_{k+1} \neq \bar{a}_{k+1}, b_{k}=\bar{b}_{k}$, then there is a unique developable passing through these two curves.

The edge of regression $E^{*}$ of this developable passes through $O$. Since $E^{*}$ and $C$ (or $\bar{C}$ ) have at $O$ a common tangent $Y=0, Z=0$ and common osculating plane $Z=0$, the application of our results of Theorems 7 and 8 to $E^{*}$ and $C$ (or $\bar{C}$ ) leads to a covariant line. But we particularly remark that the developable of Theorem 9 is tangent to $Z=0$ along

$$
Z=0, \quad \frac{X}{b_{k+2}-b_{k+2}}+\frac{Y}{3 b_{0}\left(a_{k+1}-\bar{a}_{k+1}\right)}=0
$$

when $b_{k+1}=b_{k+1}, b_{k+2} \neq b_{k+2}$.

A direct analogy of Chang's correspondence ${ }^{13} F$ is that the osculating planes of two space curves at their corresponding points pass through one and the same point on the common tangent of these two curves. In general, there are two ways to generalize the correspondence $F$. Firstly, let the points $P$ and $\bar{P}$ of the curves $C$ and $\bar{C}$ in $N$-dimensional space so correspond that a prescribed $(N-1-2 \mu)$-dimensional space $(2 \mu+1<N)$ and the osculating linear spaces $S_{\mu}(P)$ and $S_{\mu}(\bar{P})$ of $C$ and $\bar{C}$ at $P$ and $\bar{P}$ belong to one and the same hyperplane, and secondly, let $P$ and $\bar{P}$ so correspond that the common part of the osculating linear spaces $S_{\nu}(P)$ and $S_{\nu}(\bar{P})$ of $\nu$ dimensions, where $2 \nu \geqq N$, are linearly dependent with a given $(2 N-2 \nu)$-dimensional space. These will be, however, established on another occasion.

National Tzekung Technical College

13 Chang, loc. cit. footnote 1. 